\title{
TAZ inhibition restores sensitivity of cisplatin via AKT/mTOR signaling in lung adenocarcinoma
}

\author{
WEI XU*, YUNYAN WEI*, YUE LI, YUAN YIN, WEIWEI YUAN, YAN YANG, \\ WEIHONG ZHAO and JIANQING WU
}

\begin{abstract}
Jiangsu Provincial Key Laboratory of Geriatrics, Department of Geriatrics, The First Affiliated Hospital of Nanjing Medical University, Nanjing, Jiangsu 210029, P.R. China
\end{abstract}

Received January 21, 2017; Accepted July 12, 2017

DOI: $10.3892 / o r .2017 .5847$

\begin{abstract}
The cisplatin-based doublet remains the foundation of treatment for the majority of patients with advanced non-small cell lung cancer (NSCLC), however, many patients exhibit acquired drug resistance. Transcriptional co-activator with PDZ binding motif (TAZ) is an oncogene in lung cancer, important for lung tumorigenesis and metastasis. Recently, it was revealed that TAZ upregulation confers resistance against a diverse range of cytotoxic agents. The present study aimed to investigate whether TAZ is involved in cisplatin sensitivity in lung adenocarcinoma. In the present study, we investigated TAZ expression in lung adenocarcinoma tissues and cell lines with western blot and RT-PCR analyses. By knocking down TAZ using short hairpin RNAs in cisplatin-resistant A549 and H460 cells, cell proliferation and apoptosis were assessed by BrdUrd labeling and flow cytometric analysis, respectively. The $\mathrm{IC}_{50}$ value of cisplatin was also assessed with CCK-8 assays. The western blotting for AKT, p-AKT, S6K and p-S6K were performed in cells with TAZ knockdown or overexpression. When the AKT/mTOR pathway was blocked in A549 and H460 with TAZ overexpression, cisplatin sensitivity was assessed by $\mathrm{IC}_{50}$ value. High expression of TAZ was found in lung adenocarcinoma tissues and cell lines, which were associated with cisplatin resistance. Knockdown of TAZ using shRNAs
\end{abstract}

Correspondence to: Professor Jianqing Wu, Jiangsu Provincial Key Laboratory of Geriatrics, Department of Geriatrics, The First Affiliated Hospital of Nanjing Medical University, 300 Guangzhou Road, Nanjing, Jiangsu 210029, P.R. China

E-mail: jwunjmu@163.com

${ }^{*}$ Contributed equally

Abbreviations: S6K, ribosomal protein S6 kinase; EGFR, epidermal growth factor receptor; CYR61, cysteine rich angiogenic inducer 61; CTGF, connective tissue growth factor; RUNX2, runt-related transcription factor 2; MyoD, class I myosin; PPAR, peroxisome proliferator-activated receptor; TTF1, transcription termination factor 1; PAX3, paired box 3

Key words: TAZ, proliferation, chemosensitivity, AKT/mTOR signaling pathway, lung adenocarcinoma confered decreasing proliferation, increasing apoptosis and enhanced cisplatin sensitivity in cisplatin-resistant cells. Additionally, TAZ knockdown decreased the AKT/mTOR pathway expression. Overexpression of TAZ increased p-AKT and p-S6K, which was inhibited by siAKT. Furthermore, we found that the inhibition of the AKT/mTOR pathway rescued the cells from cisplatin resistance caused by TAZ overexpression. Our data revealed that TAZ inhibition restores sensitivity of cisplatin in lung adenocarcinoma, which was, at least in part, AKT/mTOR signaling pathway-dependent. TAZ may be a potent therapeutic target for NSCLC in combination with conventional chemotherapy.

\section{Introduction}

Non-small cell lung cancer (NSCLC) is the leading cause of cancer-related deaths worldwide. The treatment of advanced NSCLC may be changing since the emergence of targeted therapy, but the cisplatin-based doublet remains the foundation of treatment for the majority of patients with advanced NSCLC (1). The resistance to chemotherapy has been a major factor affecting the therapeutic efficacy in the treatment of lung cancer. To overcome the resistance, second line or combination chemotherapy regimens have been used, but the overall survival benefits of various chemotherapies in NSCLC are not yet satisfactory (2).

Transcriptional co-activator with PDZ binding motif (TAZ), also known as WW domain-containing transcription regulator-1, is a key transducer of the Hippo pathway. This pathway is an essential regulator of organ size, stem cell maintenance, and tumorigenesis. TAZ acts mainly through the TEAD family of transcription factors to stimulate expression of genes that promote cell proliferation and control organ size. TAZ has also been suggested to be involved in other biological processes, including mesenchymal stem cell differentiation (3), self-renewal of human embryonic stem cells (4) and mechanotransduction (5). In addition, TAZ may crosstalk with different signaling pathways in different cell types (6).

Growing evidence has revealed that TAZ is an oncogene in lung cancer, important for lung tumorigenesis and metastasis (7). Clinical lung cancer patient studies also reported that TAZ is overexpressed in over 60\% of NSCLC and its expression is significantly associated with adenocarcinoma, 
poor differentiation, metastasis and poor prognosis and survival (8). Recent studies have consistently suggested that upregulation of TAZ and/or its paralog YAP confers resistance against a diverse range of cytotoxic agents such as anti-tubulin, antimetabolite and DNA-damaging agents (9). TAZ overexpression in breast cancer cells has been shown to induce resistance to taxol and doxorubicin, and upregulation of its target genes CYR61 and CTGF appears to contribute to resistance development (10). YAP has been reported to promote resistance to cisplatin in oral squamous cell carcinoma (11), urothelial cell carcinoma (12) and ovarian cancer (13). In the present study, we provided the first evidence that TAZ is also involved in cisplatin sensitivity in lung adenocarcinoma.

\section{Materials and methods}

Reagents and antibodies. Cisplatin (APP Pharmaceuticals LLC, Schaumburg, IL, USA) stock solution (3.3 mmol/l) prepared in sterile water was provided by the outpatient pharmacy, The First Affiliated Hospital of Nanjing Medical University (Jiangsu, China). TAZ, AKT, p-AKT (Ser473/Thr308) and S6K antibodies were obtained from Cell Signaling Technology, Inc. (Danvers, MA, USA). The phospho-p70 S6 kinase (Thr389/412) antibody was purchased from Affinity Biologicals (Ancaster, Canada). The GAPDH antibody was obtained from Santa Cruz Biotechnology, Inc. (Santa Cruz, Califirnia, USA).

Cell culture. Human bronchial epithelial cell line $16 \mathrm{HBE}$ and lung adenocarcinoma cell lines A549 and H460 were purchased from the Cell Resource Center (Shanghai Institutes for Biological Sciences, Shanghai, China). The cisplatin-resistant A549 (A549/DDP) and H460 (H460/DDP) cell lines were kindly provided by Professor Zhou at the Shanghai Pulmonary Hospital. All cell lines were maintained in RPMI-1640 medium containing $10 \%$ fetal bovine serum (FBS) (both from Life Technologies, Inc., Gaithersburg, MD, USA), $100 \mathrm{U} / \mathrm{ml}$ penicillin, $100 \mathrm{U} / \mathrm{ml}$ streptomycin, $2 \mathrm{mM}$ glutamine in a humidified atmosphere with $5 \% \mathrm{CO}_{2}$ at $37^{\circ} \mathrm{C}$. To maintain drug resistance, A549/DDP and H460/DDP cells were grown in RPMI-1640 medium containing $2 \mathrm{mg} / \mathrm{ml} \mathrm{DDP}$, and then in DDP-free RPMI-1640 medium two days before experiments.

Reverse transcription PCR (RT-PCR). Total RNA was extracted from cells with TRIzol reagent (Invitrogen, San Diego, CA, USA) according to the manufacturer's instructions and subjected to cDNA synthesis and PCR. The primers used were as follows: The specific primers were as follows: TAZ forward, 5'-AGTACCCTGAGCCAGCAGAA-3' and reverse, 5'-GATTCTCTGAAGCCGCAGTT-3'; GAPDH forward, 5'-GGAGCCAAAAGGGTCATCAT-3' and reverse, 5'-GTGATGGCATGGACTGTGGT-3'.

Western blot analysis. Cells were lysed in RIPA buffer (50 mmol/1 Tris-HCl buffer, $\mathrm{pH} \mathrm{7.4,} 150 \mathrm{mmol} / \mathrm{l} \mathrm{NaCl}, 1 \%$ Triton X-100, $1 \%$ sodium deoxycholate and $0.1 \%$ SDS) supplemented with 1X Halt protease inhibitor cocktail and 1X Halt phosphatase inhibitor cocktail (Pierce Biotechnology, Inc., Rockford, IL, USA). The proteins were separated and western blot analysis was carried out as previously described (14). The target protein was visualized by chemiluminescence (Denville Scientific, Inc., Metuchen, NJ, USA).

Immunohistochemistry (IHC). Formalin-fixed paraffinembedded tumor tissues were obtained from the The First Affiliated Hospital of Nanjing Medical University. IHC was performed using the protocol as previously described (14), using the TAZ antibody (Cell Signaling Technology, Inc., Danvers, MA, USA). Immunostaining was classified on the basis of the intensity of staining (no staining, 0 ; weak, 1; moderate, 2; and strong, 3 points). A final IHC score was obtained by multiplying the intensity score and extent (\%) of stained cells. The Institutional Review Board (IRB) approved the present study.

Cell Counting Kit-8 assay. Cell Counting Kit-8 (CCK-8) assay (Beyotime Institute of Biotechnology, Beijing, China) was used to detect cell proliferation. According to the manufacturer's instructions, the absorbance was measured at a wavelength of $450 \mathrm{~nm}$ using Bio-Tek microplate reader ELx800 (Bio-Tek, Winooski, VT, USA) every $24 \mathrm{~h}$ after transfection. The half maximal inhibitory concentration $\left(\mathrm{IC}_{50}\right)$ of lung adenocarcinoma cells were also determined using the CCK- 8 assay.

BrdUrd-labeling. Cells were labeled with $10 \mathrm{mmol} / 1$ bromodeoxyuridine (BrdUrd; Sigma-Aldrich, St. Louis, MO, USA) in growth medium for $12 \mathrm{~h}$ at $37^{\circ} \mathrm{C}$. BrdUrd-labeled DNA was detected with mouse monoclonal anti-BrdUrd (Ruibo Biotech, Guangzhou, China) according to the manufacturer's protocol. Cells were examined under Olympus IX-71 (Olympus, Tokyo, Japan) inverted microscope and photographed.

Flow cytometry. Cells were harvested and gently disaggregated to a single cell suspension. Staining was made according to the manufacturer's protocol. The rate of apoptosis induced by anticancer regimens was analyzed by flow cytometry using an Annexin V-FITC/PI kit (BD Biosciences, San Diego, CA, USA) following the manufacturer's instructions.

Plasmid and transfection. The target sequences of shTAZ1 and shTAZ2 were: GCGATGAATCAGCCTCTGAAT and AGGTACTTCCTCAATCACA. Cells reaching 80\% confluence were transfected with pGPU6-shTAZ in the presence of Lipofectamine 2000 (Invitrogen). The plasmid of pEX2-TAZ and negative control (pEX2), AKT-siRNA and siRNA negative control (siNC) were purchased from GenePharma (Shanghai, China). The sequence of siAKT and siNC were: 5'-GAACAAUCCGAUUCACGUATT-3' and 5'-UUCUCCG AACGUGUCACGUTT-3', respectively.

Statistical analysis. The data are expressed as the mean \pm SD; Student's t-tests and Chi-square tests were used to determine the significance of differences in multiple comparisons. All data were analyzed with SPSS statistical software (version 13.0; SPSS, Inc., Chicago, IL, USA) and a P-value of $<0.05$ was considered statistically significant.

\section{Results}

High expression of TAZ in lung adenocarcinoma. We investigated TAZ expression in 10 cases of lung adenocarcinoma 


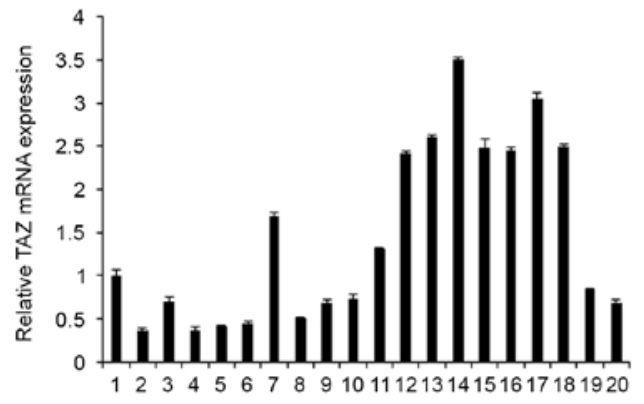

D

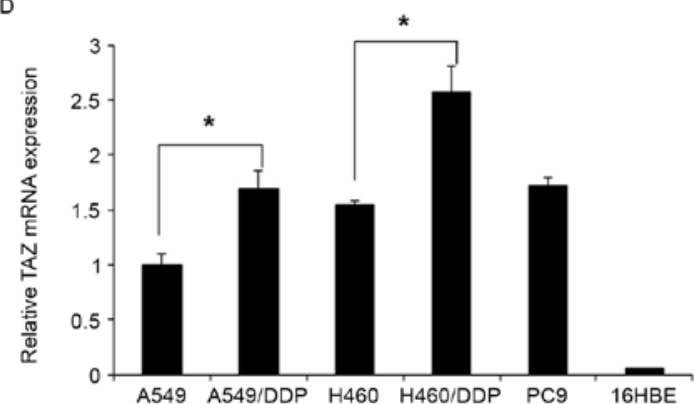

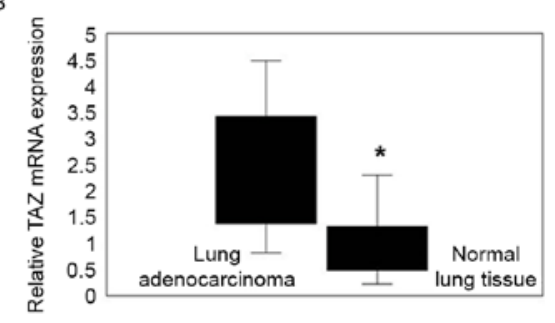

c

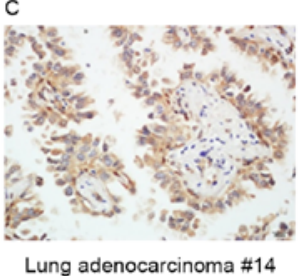

By IHC $(\times 400)$
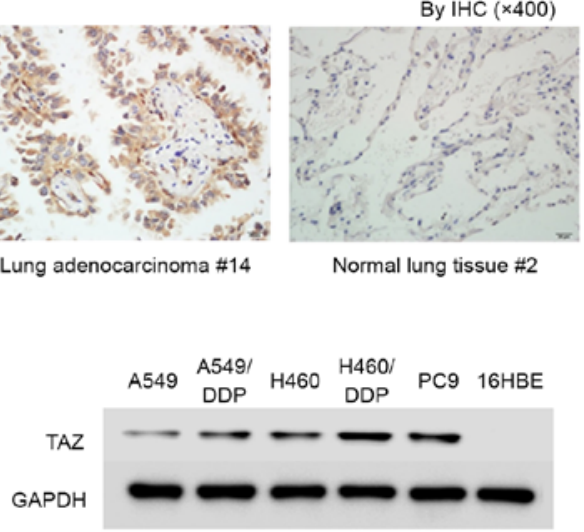

Figure 1. TAZ expression in lung adenocarcinoma tissue and cell lines. (A and B) RT-PCR analysis of TAZ expression in 10 cases of lung adenocarcinoma tissue and 10 cases of normal lung tissue, normalized against GAPDH. (C) Immunohistochemistry staining of TAZ in lung adenocarcinoma tissue and normal lung tissue. TAZ-positive expression in lung adenocarcinoma, staining in both cytoplasm and nucleus of tumor cells (magnification, $\mathrm{x} 400$ ). (D) RT-PCR analysis of TAZ expression in a panel of 5 human lung adenocarcinoma cell lines (A549, A549/DDP, H460, H460/DDP and PC9) and non-neoplastic bronchial epithelial cell line (16HBE), normalized against GAPDH. (E) Western blot analysis of TAZ protein levels in the human lung adenocarcinoma cell lines and the bronchial epithelial cell line. GAPDH served as an internal reference. Detection was performed in triplicate and data were presented as the mean \pm SEM; ${ }^{*} \mathrm{P}<0.05$. TAZ, transcriptional co-activator with PDZ binding motif.

tissue and 10 cases of normal lung tissue by qRT-PCR (Fig. 1A) and IHC. TAZ was expressed at levels 3.15 times higher in lung adenocarcinoma than those in normal lung tissue (Fig. 1B). TAZ-positive expression in tumor cells was observed in 8 out of 10 lung adenocarcinoma samples. Both cytoplasmic and nuclear TAZ was present in lung adenocarcinoma tissue. TAZ overexpression was not detected in non-neoplastic bronchial or alveolar epithelial cells (Fig. 1C).

We examined the TAZ expression in lung cell lines by qRT-PCR (Fig. 1D) and western blotting (Fig. 1E). Specifically, no TAZ expression was observed in non-tumorigenic human bronchial epithelial cell line (16HBE), whereas overexpression of TAZ was observed in tumorigenic lung adenocarcinoma cell lines (A549, A549/DDP, A549/TR, H460 and PC9). The relative mRNA level of TAZ in A549/DDP and H460/DDP cells was $\sim 1.7$ and 2.58 times higher than that in A549 and H460 cells, respectively (Fig. 1D). Western blot assay revealed the same trend in the protein expression of TAZ in cell lines.

TAZ knockdown regulates the proliferation, apoptosis and cisplatin sensitivity in cisplatin-resistant cells. To further investigate the role of TAZ in the resistance of the cells to chemotherapy, TAZ knockdown was performed in A549/DDP and H460/DDP cells with specific shRNAs (Fig. 2A and B). The results demonstrated that the shTAZ2 exhibited the best interference efficiency. In the present study, shTAZ2 was employed in some of the following experiments. To elucidate the effect of TAZ on cell proliferation, shTAZ2 was transfected into A549/DDP and H460/DDP cells. After $48 \mathrm{~h}$ of transfection, the rate of cell proliferation and growth was significantly decreased in both A549/DDP and H460/DDP cells assessed by BrdU incorporation and CCK-8 assay. The mean percentage of positive proliferative cells of the shTAZ2 group decreased by $40 \%(\mathrm{P}<0.05)$ in the A549/DDP cells and $\sim 50 \%$ in the H460/DDP cells. The result of the CCK-8 assay revealed that the number of cells decreased by $40.0 \%$ in the A549/DDP cells (Fig. 2C and D) and 29.8\% in the H460/DDP cells (Fig. 2E and F) after transfection on the 6th day.

We subsequently explored the effect of TAZ knockdown on cell apoptosis using by Annexin V-FITC/PI double staining. As shown in Fig. $3 \mathrm{~A}$ and $\mathrm{B}$, the apoptosis rate of the shTAZ2 group increased by $70 \%(\mathrm{P}<0.05)$ in the A549/DDP cells and 46\% $(\mathrm{P}<0.05)$ in the H460/DDP cells, indicating that TAZ knockdown induces apoptosis in cisplatin-resistant lung adenocarcinoma cells.

Next, in order to assess the effect of TAZ knockdown on the sensitivity of the resistant cells to cisplatin a CCK-8 assay was employed to examine the $\mathrm{IC}_{50}$ value of A549/DDP and H460/DDP cells (Fig. 3C and D). The results revealed that the $\mathrm{IC}_{50}$ value of the shRNA-transfected cells had a pronounced decrease in the $\mathrm{IC}_{50}$ value as compared to the negative control group. The mean $\mathrm{IC}_{50}$ value of the A549/DDP cells transfected shTAZ2 was $27.83 \pm 4.23 \mu \mathrm{M}$ vs. $48.92 \pm 6.62 \mu \mathrm{M}$ in the negative control group. In the H460/DDP cells, the mean $\mathrm{IC}_{50}$ value for the shTAZ2-transfection group was $30.53 \pm 5.76 \mu \mathrm{M}$ vs. the negative control group which was $58.62 \pm 7.54 \mu \mathrm{M}$.

TAZ regulates the AKT/mTOR signaling pathway in lung adenocarcinoma cells. Previous studies demonstrated that TAZ affected cell chemosensitivity by phosphorylation of 

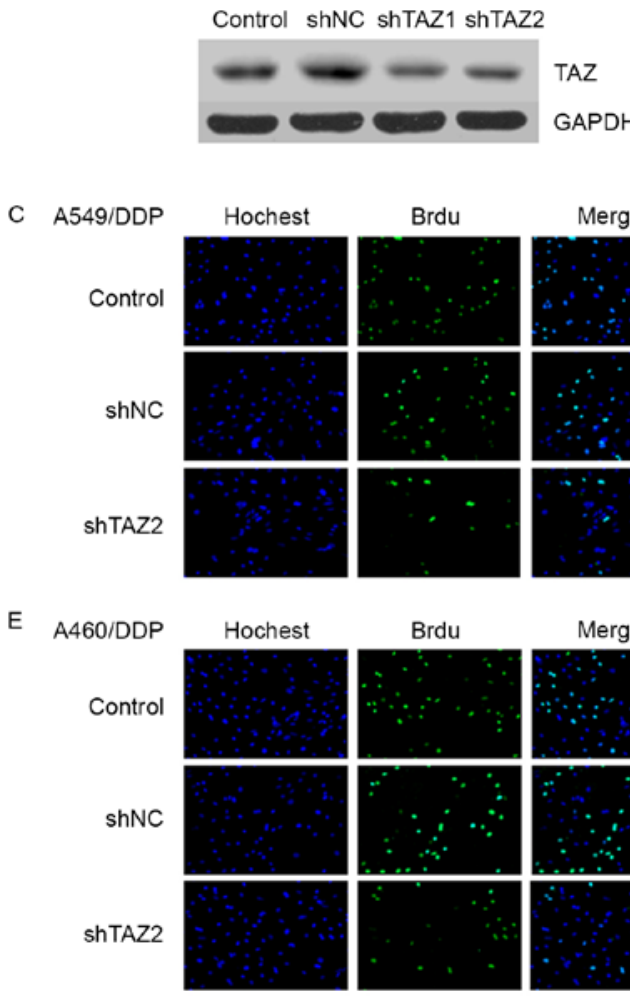

Brdu
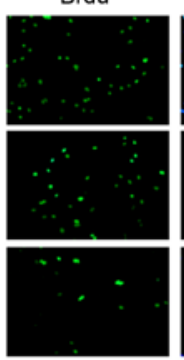

Brdu

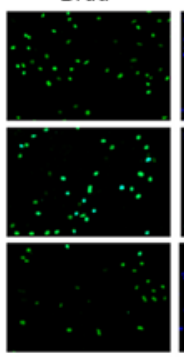

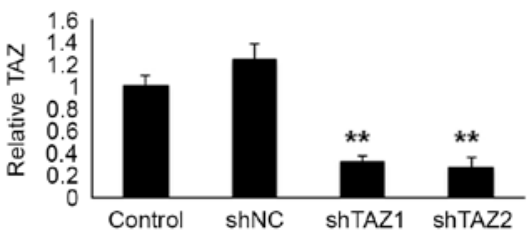

D

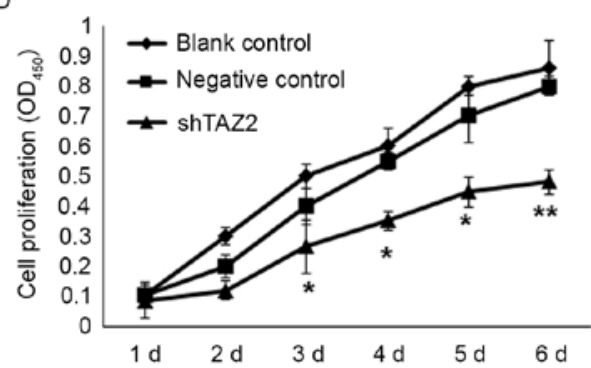

F
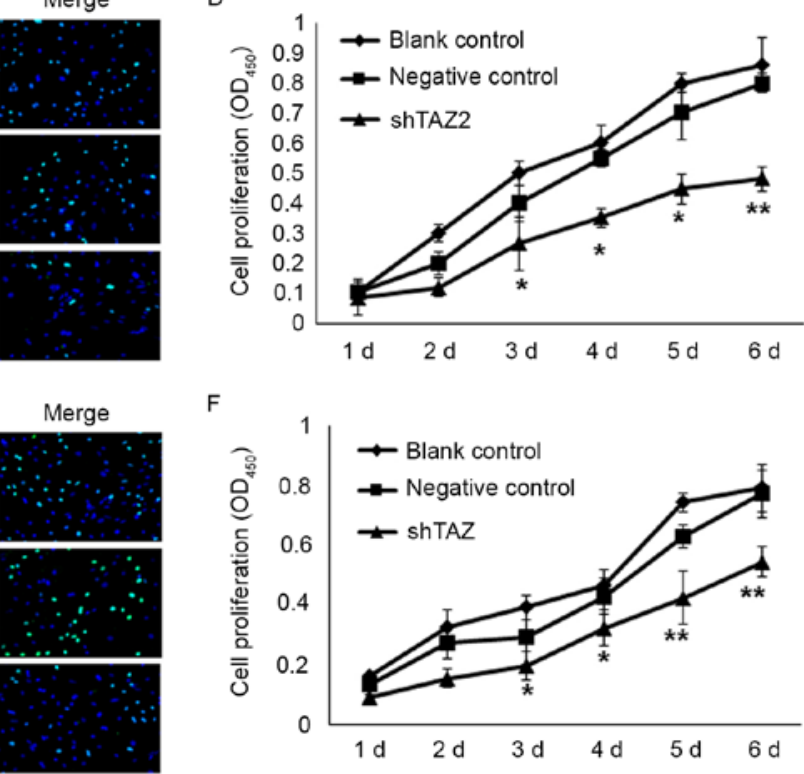

Figure 2. TAZ knockdown regulates the proliferation in cisplatin-resistant cells. (A and B) Representative western blot and RT-PCR analyses of TAZ downregulation efficiency after shRNA-transfection for $48 \mathrm{~h}$. shNC, shRNA negative control. (C and D) Representative BrdU analysis of cell proliferation after shTAZ2- or shNC-transfection in A549/DDP cells for $48 \mathrm{~h}$. DAPI (blue) was used to stain the nucleus and BrdU (green) showed the incorporated cells. A549/DDP cell proliferation was assessed by the CCK-8 assay at the indicated time-points. (E and F) Representative BrdU analysis of cell proliferation after shTAZ2- or shNC-transfection in H460/DDP cells for $48 \mathrm{~h}$. H460/DDP cell growth was assessed by the CCK-8 assay. All the results are shown as the mean \pm SEM of three independent experiments; ${ }^{*} \mathrm{P}<0.05,{ }^{* *} \mathrm{P}<0.01$. TAZ, transcriptional co-activator with PDZ binding motif.
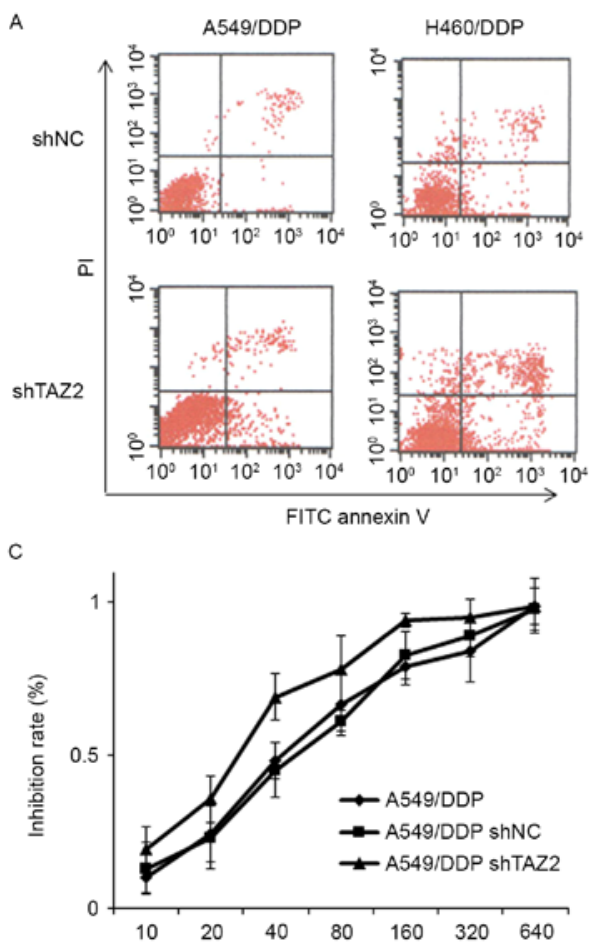

B

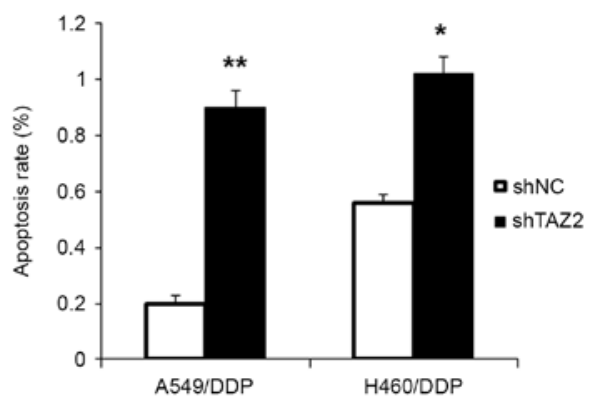

D

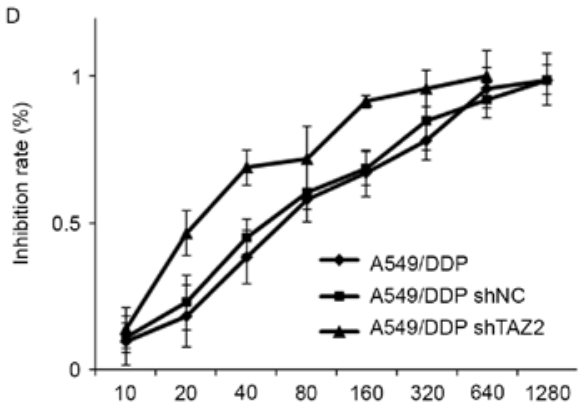

Figure 3. TAZ knockdown regulates apoptosis and cisplatin sensitivity in cisplatin-resistant cells. (A) Representative flow cytometric analysis of cell apoptosis co-stained with Annexin V/PI after shTAZ- or shNC-transfection for $48 \mathrm{~h}$. The right lower quadrant in the dot plot represents the early stage of apoptotic cells. (B) Quantitative analysis of the percentage of apoptotic cells in the early stage. (C) A549/DDP and (D) H460/DDP cells were transfected with TAZ-specific shRNA or shNC as a negative control. A CCK-8 assay revealed a decreased $\mathrm{IC}_{50}$ of cells transfected with shTAZ2. All the results are shown as the mean \pm SEM of three independent experiments; ${ }^{*} \mathrm{P}<0.05,{ }^{* *} \mathrm{P}<0.01$. TAZ, transcriptional co-activator with PDZ binding motif. 


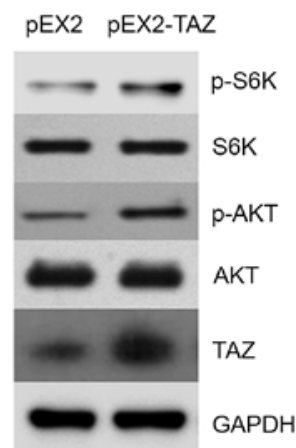

$\mathrm{C}$

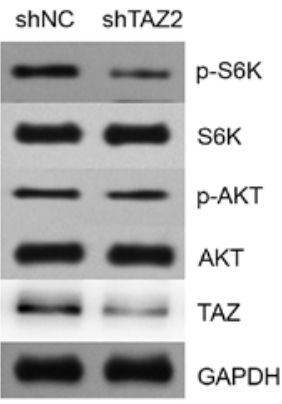

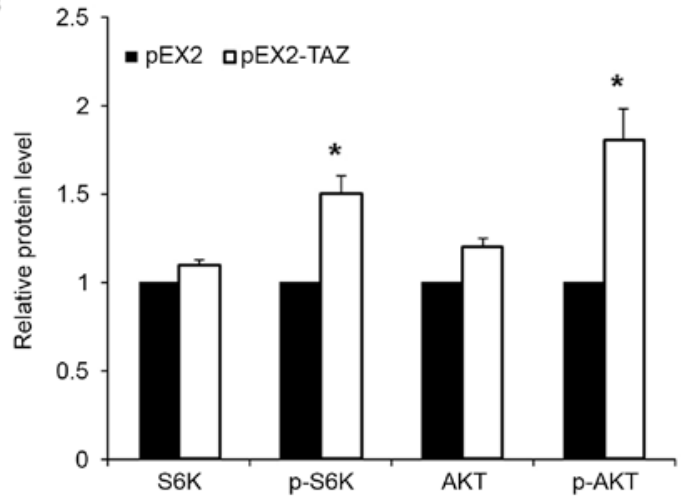

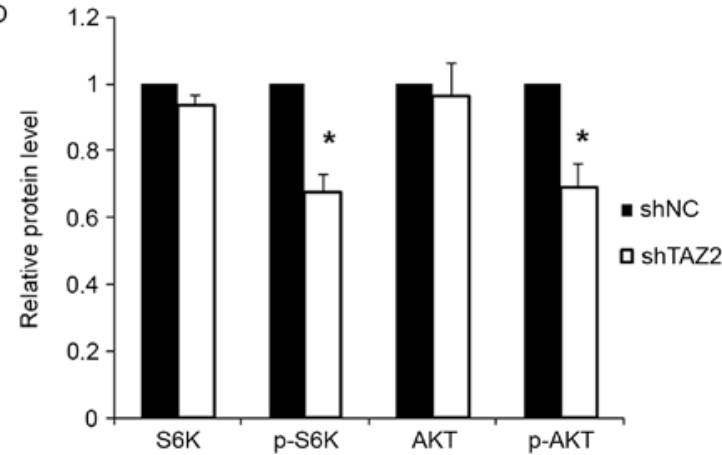

Figure 4. TAZ regulates the AKT/mTOR signaling pathway in lung adenocarcinoma cells. (A) A549 cells transfected with pEX2-TAZ were treated with siAKT or siNC. Western blot analysis of total and phosphorylation levels of AKT and S6K after treatment. (B) Quantitative analysis of total and phosphorylation levels of AKT and S6K after TAZ overexpression. (C) AKT, p-AKT, S6K and p-S6K were examined in TAZ-shRNA transfected A549/DDP cells via western blot assay. (D) Quantitative analysis of AKT, p-AKT, S6K and p-S6K after TAZ knockdown. Detection was performed in triplicate and data are presented as the mean $\pm \mathrm{SEM} ;{ }^{*} \mathrm{P}<0.05$. TAZ, transcriptional co-activator with PDZ binding motif; S6K, ribosomal protein S6 kinase.

A

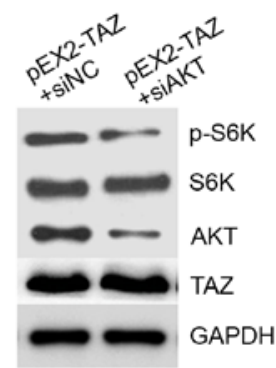

C

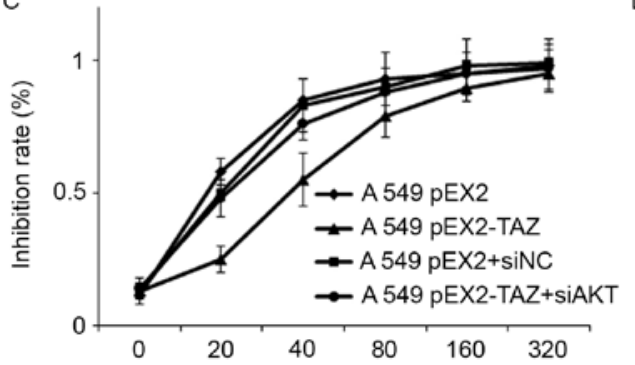

B
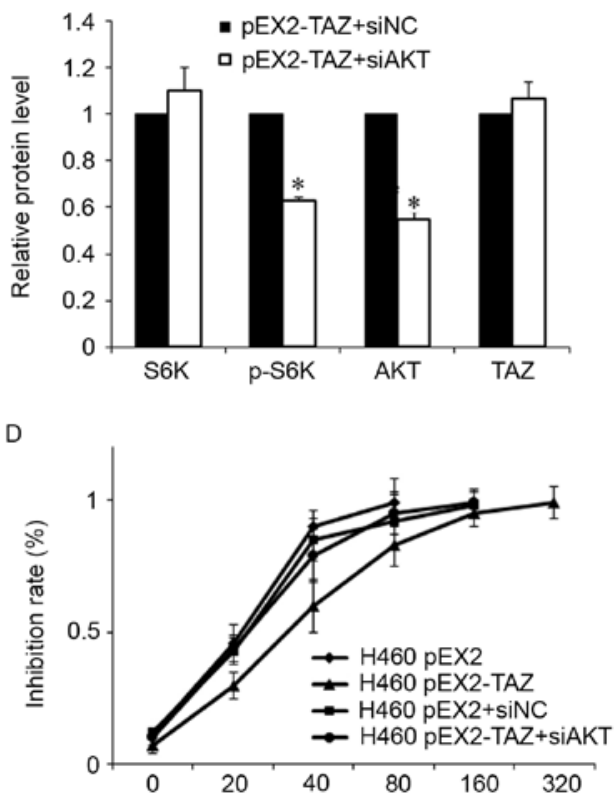

Figure 5. Inhibition of AKT/mTOR signaling rescues cisplatin sensitivity decreased by TAZ overexpression. (A) A549 cells transfected with pEX2-TAZ were treated with siAKT or siNC. Western blot analysis of total and phosphorylation levels of S6K after pEX2-TAZ and siAKT treatment. (B) Quantitative analysis of total and phosphorylation levels of AKT and S6K after TAZ overexpression and siAKT treatment. (C) A549 and (D) H460 cells transfected with pEX2-TAZ were treated with siAKT or siNC. A CCK-8 assay was performed to assess the $\mathrm{IC}_{50}$ values. Detection was performed in triplicate and data are presented as the mean $\pm \mathrm{SEM} ;{ }^{*} \mathrm{P}<0.05$. TAZ, transcriptional co-activator with PDZ binding motif; S6K, ribosomal protein S6 kinase.

AKT. To detect whether TAZ affects lung adenocarcinoma cell sensitivity to cisplatin by regulating the AKT/mTOR signaling pathway, we firstly overexpressed TAZ in cancer cells. As shown in Fig. 4A, the protein expression of TAZ in A549 cell 
lines were markedly increased after transfection. Moreover, the expression of total AKT, phosphorylated AKT (p-AKT), total S6K and phosphorylated S6K (p-S6K) were examined in transfected cells. Cells with TAZ overexpression exhibited increased expression of $\mathrm{p}-\mathrm{AKT}$ and $\mathrm{p}-\mathrm{S} 6 \mathrm{~K}$ when compared with the control group (Fig. 4A and B).

Furthermore, the expression of AKT, p-AKT, S6K and p-S6K were examined in TAZ shRNA-transfected cells. Western blot assay revealed that TAZ knockdown led to the significant decrease in the expression of p-AKT and p-S6K (Fig. 4C and D).

TAZ affects the sensitivity of cisplatin via AKT/mTOR signaling. We used AKT-specific siRNA (siAKT) to downregulate the expression of AKT and to evaluate whether the effects of TAZ on lung adenocarcinoma cells depended on the AKT/mTOR signaling pathway. As is shown in Fig. 5A, the level of p-S6K was markedly decreased after siAKT transfection, in line with the previous experiments. Meanwhile, the increased level of $\mathrm{p}$-S6K-induced by TAZ overexpression was partially abolished by AKT downregulation (Fig. 5A and B).

Furthermore, AKT downregulation restored the sensitivity to cisplatin both in A549 and H460 cells (Fig. 5B and C). The increased $\mathrm{IC}_{50}$ value induced by $\mathrm{TAZ}$ overexpression was eliminated by siAKT.

\section{Discussion}

Although platinum-based chemotherapy alone or in combination with other drugs has been used as a first-line therapy in patients with advanced NSCLC, resistance to cisplatin, a platinum compound, unfortunately, remains a major clinical problem. The mechanisms of cisplatin resistance are not fully understood, but they are believed to be multifactorial in nature (15). They include insufficient DNA binding, increased detoxification and DNA repair, dysregulated expression of transporters, and altered expression and activation of genes involved in cell death pathways, such as p53, Bcl-2 and AKT/mTOR. Among these pathways, the activation of the AKT/mTOR pathway plays an important role in cisplatin resistance (16).

TAZ has emerged as a key player in organ growth and tumorigenesis (17). It is tightly regulated in the hippo pathway in a dependent and independent manner in response to a wide range of extracellular and intrinsic signals, including cell density, cell polarity, F-actin-related mechanical stress, ligands of $\mathrm{G}$ protein-coupled receptors (GPCRs), cellular energy status, hypoxia and osmotic stress (18). TAZ has been implicated to interact with multiple transcription factors including RUNX2, MyoD, PPAR, TTF1, PAX3, PAX8, Smads and TEADs (19).

Recent studies have consistently suggested that TAZ/YAP upregulation confers resistance against a diverse range of cytotoxic agents (9). The increased levels of TAZ were found in human breast cancer cells which were responsible for their resistance to Taxol though the TAZ-TEAD-Cyr61/CTGF signaling pathway (10). Gene-expression profile studies comparing metastagenic and non-metastagenic cells identified TAZ as a central mediator of patient-derived breast cancer stem cell lines metastatic ability involved in their chemoresistance and tumorigenic potential (20). Cisplatin-resistant oral squamous cancer cell lines exhibited decreased phospho-YAP and increased nuclear YAP (11). Furthermore, YAP overexpression protected while YAP knockdown sensitized urothelial carcinoma cells to chemotherapy and radiation effects via increased accumulation of DNA damage and apoptosis. Pharmacological YAP inhibition with verteporfin inhibited tumor cell proliferation and restored sensitivity to cisplatin (12). Moreover, YAP signaling may regulate cisplatin resistance in ovarian cancer cells by increasing cellular autophagic flux (13).

We previously revealed that TAZ is upregulated in lung adenocarcinoma cells with the EGFR T790M mutation, and TAZ depletion inhibits tumorigenicity, epithelial mesenchymal transition, migration, invasion of gefitinib-resistant NSCLC cells and sensitizes their response to gefitinib (14). In the present study, we found that TAZ was highly expressed in lung adenocarcinoma tissues and cell lines, particularly in cisplatin-resistant cells. Knockdown of TAZ confers decreased proliferation, increased apoptosis and cisplatin sensitivity in cisplatin-resistant cells. We also elucidated the possible signaling transduction mechanism of TAZ on the cisplatin sensitivity in human lung adenocarcinoma and affirmed that TAZ knockdown decreased the AKT/mTOR pathway expression. Overexpression of TAZ increased $\mathrm{p}-\mathrm{AKT}$ and p-S6K, which was inhibited by siAKT. Furthermore, we found the that inhibition of the AKT/mTOR pathway rescued the cells from chemoresistance caused by TAZ overexpression. These results demonstrated that chemosensitivity regulation of TAZ was, at least in part, AKT/mTOR signaling pathway-dependent.

In summary, in the present study our data indicated that inhibition of the transcription co-activator TAZ was linked to cisplatin sensitivity in lung adenocarcinoma for the first time, which was partly dependent on the AKT/mTOR signaling pathway. Thus, TAZ may be a potent therapeutic target for NSCLC in combination with conventional chemotherapy.

\section{Acknowledgements}

The present study was funded by Jiangsu Provincial Key Discipline of Medicine (ZDXKA2016003), the Priority Academic Program Development of Jiangsu Higher Education Institutions (Jiangsu, China), and was also supported by grants from the International Science and Technology Cooperation Program of China (no. 2014DFA31940), the National Natural Science Foundation of China (Beijing, China; nos. 81302014, 81572259 and 81402489), the Six Talent Peaks Project (Jiangsu, China; no. 2015-WSN-038) and the 333 High-level Personnel Training Project of Jiangsu Province.

\section{References}

1. Fennell DA, Summers Y, Cadranel J, Benepal T, Christoph DC, Lal R, Das M, Maxwell F, Visseren-Grul C and Ferry D: Cisplatin in the modern era: The backbone of first-line chemotherapy for non-small cell lung cancer. Cancer Treat Rev 44: 42-50, 2016.

2. Shi Y and Sun Y: Medical management of lung cancer: Experience in China. Thorac Cancer 6: 10-16, 2015.

3. Hong JH, Hwang ES, McManus MT, Amsterdam A, Tian Y, Kalmukova R, Mueller E, Benjamin T, Spiegelman BM, Sharp PA, et al: TAZ, a transcriptional modulator of mesenchymal stem cell differentiation. Science 309: 1074-1078, 2005. 
4. Zhao B, Tumaneng K and Guan KL: The Hippo pathway in organ size control, tissue regeneration and stem cell self-renewal. Nat Cell Biol 13: 877-883, 2011.

5. Dupont S, Morsut L,Aragona M,EnzoE,Giulitti S, Cordenonsi M, Zanconato F, Le Digabel J, Forcato M, Bicciato S, et al: Role of YAP/TAZ in mechanotransduction. Nature 474: 179-183, 2011.

6. Zhang K, Qi HX, Hu ZM, Chang YN, Shi ZM, Han XH, Han YW, Zhang RX, Zhang Z, Chen T, et al: YAP and TAZ take center stage in cancer. Biochemistry 54: 6555-6566, 2015.

7. Zhou Z, Hao Y, Liu N, Raptis L, Tsao MS and Yang X: TAZ is a novel oncogene in non-small cell lung cancer. Oncogene 30 : 2181-2186, 2011

8. Xie M, Zhang L, He CS, Hou JH, Lin SX, Hu ZH, Xu F and Zhao HY: Prognostic significance of TAZ expression in resected non-small cell lung cancer. J Thorac Oncol 7: 799-807, 2012.

9. Kim MH and Kim J: Role of YAP/TAZ transcriptional regulators in resistance to anti-cancer therapies. Cell Mol Life Sci 74 $1457-1474,2017$.

10. Lai D, Ho KC, Hao Y and Yang X: Taxol resistance in breast cancer cells is mediated by the hippo pathway component TAZ and its downstream transcriptional targets Cyr61 and CTGF. Cancer Res 71: 2728-2738, 2011.

11. Yoshikawa K, Noguchi K, Nakano Y, Yamamura M, Takaoka K, Hashimoto-Tamaoki T and Kishimoto H: The Hippo pathway transcriptional co-activator, YAP, confers resistance to cisplatin in human oral squamous cell carcinoma. Int $\mathrm{J}$ Oncol 46: 2364-2370, 2015.

12. Ciamporcero E, Shen H, Ramakrishnan S, Yu Ku S, Chintala S, Shen L, Adelaiye R, Miles KM, Ullio C, Pizzimenti S, et al: YAP activation protects urothelial cell carcinoma from treatmentinduced DNA damage. Oncogene 35: 1541-1553, 2016.
13. Xiao L, Shi XY, Zhang Y, Zhu Y, Zhu L, Tian W, Zhu BK and Wei ZL: YAP induces cisplatin resistance through activation of autophagy in human ovarian carcinoma cells. Onco Targets Ther 9: 1105-1114, 2016.

14. Xu W, Wei Y, Wu S, Wang Y, Wang Z, Sun Y, Cheng SY and Wu J: Up-regulation of the Hippo pathway effector TAZ renders lung adenocarcinoma cells harboring EGFR-T790M mutation resistant to gefitinib. Cell Biosci 5: 7, 2015.

15. Amable L: Cisplatin resistance and opportunities for precision medicine. Pharmacol Res 106: 27-36, 2016.

16. Xu JL, Wang ZW, Hu LM, Yin ZQ, Huang MD, Hu ZB, Shen HB and Shu YQ: Genetic variants in the PI3K/PTEN/AKT/mTOR pathway predict platinum-based chemotherapy response of advanced non-small cell lung cancers in a Chinese population. Asian Pac J Cancer Prev 13: 2157-2162, 2012.

17. Noguchi S, Saito A, Horie M, Mikami Y, Suzuki HI, Morishita Y, Ohshima M, Abiko Y, Mattsson JS, König H, et al: An integrative analysis of the tumorigenic role of TAZ in human non-small cell lung cancer. Clin Cancer Res 20: 4660-4672, 2014.

18. Moroishi T, Hansen CG and Guan KL: The emerging roles of YAP and TAZ in cancer. Nat Rev Cancer 15: 73-79, 2015.

19. Guo L and Teng L: YAP/TAZ for cancer therapy: Opportunities and challenges (Review). Int J Oncol 46: 1444-1452, 2015.

20. Bartucci M, Dattilo R, Moriconi C, Pagliuca A, Mottolese M, Federici G, Benedetto AD, Todaro M, Stassi G, Sperati F, et al: TAZ is required for metastatic activity and chemoresistance of breast cancer stem cells. Oncogene 34: 681-690, 2015. 